\title{
Deformation of Kupershmidt operators and Kupershmidt-Nijenhuis structures of a Malcev algebra
}

\author{
Sami Mabrouk (iD \\ University of Gafsa, Faculty of Sciences Gafsa, 2112 Gafsa, Tunisia
}

\begin{abstract}
The aim of this paper is to study infinitesimal deformations of a Malcev algebra with a representation and introduce the notion of Nijenhuis pair, which gives a trivial deformation of a Malcev algebra with a representation. We introduce the notion of Kupershmidt-(dual)Nijenhuis structure on a Malcev algebra with a representation. Furthermore, we show that a Kupershmidt-(dual-)Nijenhuis structure gives rise to a hierarchy of Kupershmidt operators. Finally, we establish a deformation theory of Kupershmidt operators in consistence with the general principles of deformation theories and introduce the notion of Nijenhuis elements.
\end{abstract}

Mathematics Subject Classification (2020). 17D05,17D10,17A30,17A36

Keywords. altenative algebra, Malcev algebra, representation, (dual-)Nijenhuis pair, Kupershmidt operator, Kupershmidt-(dual-)Nijenhuis structure

\section{Introduction}

Malcev algebras were introduced by Malcev [23], who called these objects MoufangLie algebras. A Malcev algebra is a non-associative algebra $A$ with an skew-symmetric multiplication [, ] that satisfies the Malcev identity

$$
J(x, y,[x, z])=[J(x, y, z), x]
$$

for all $x, y, z \in A$, where $J(x, y, z)=[[x, y], z]+[[z, x], y]+[[y, z], x]$ is the Jacobiator. In particular, Lie algebras are examples of Malcev algebras. Malcev algebras play an important role in the geometry of smooth loops. Just as the tangent algebra of a Lie group is a Lie algebra, the tangent algebra of a locally analytic Moufang loop is a Malcev algebra $[17,20,23,25,31]$. The reader is referred to $[16,24,29]$ for discussions about the relationships between Malcev algebras, exceptional Lie algebras and physics.

Closely related to Malcev algebras are alternative algebras. An alternative algebra is an algebra whose associator is an alternating function. In particular, all associative algebras are alternative, but there are plenty of non-associative alternative algebras, such as the octonions. Roughly speaking, alternative algebras are related to Malcev algebras as associative algebras are related to Lie algebras. Indeed, as Malcev observed in [23], every alternative algebra $A$ is Malcev-admissible, i.e., the commutator algebra $A^{-}$is a Malcev

Email address: mabrouksami00@yahoo.fr

Received: 21.04.2021; Accepted: 09.09.2021 
algebra. There are many Malcev-admissible algebras that are not alternative; see, e.g., [24].

The deformation of algebraic structures began with the seminal work of Gerstenhaber [11-14] for associative algebras and followed by its extension to Lie algebras by Nijenhuis and Richardson [26,28]. Deformations of other algebraic structures such as pre-Lie and Malcev algebras have also been developed in [6] and [27] respectively. In general, deformation theory was developed for binary quadratic operads by Balavoine [4]. For more general operads we refer the reader to $[10,18,21]$. Nijenhuis operators also play an important role in deformation theories due to their relationship with trivial infinitesimal deformations. There are interesting applications of Nijenhuis operators such as constructing biHamiltonian systems to study the integrability of nonlinear evolution equations $[7,9]$.

Baxter introduced the concept of a Rota-Baxter algebra for associative algebras [5] in his study of fluctuation theory in probability. There has been found many applications in recent years, including the algebraic approach of Connes-Kreimer [8] to renormalization of perturbative quantum field theory, tridendriform algebras [3], quantum analogue of Poisson geometry [32], twisting on associative algebras [33]. In Lie algebra context, a Rota-Baxter operator of weight zero was introduced independently in the 1980s as the operator form of the classical Yang-Baxter equation, named after the physicists C.-N. Yang and R. Baxter. See the book [15] for more details. Moreover, Kupershmidt restudied the classical Yang-Baxter equation [19]. Note that a Rota-Baxter operator given by SemonovTian-Shansky is just a Kupershmidt operator on a Lie algebra with respect to the adjoint representation $(\mathfrak{g}$;ad) and an $r$-matrix is a Kupershmidt operator on a Lie algebra with respect to the coadjoint representation $\left(\mathfrak{g}^{*} ; \mathrm{ad}^{*}\right)$. Moreover, the notion of an extended O-operator was introduced by Bai, Guo and $\mathrm{Ni}$ in [1,2] and plays an important role in the study of nonabelian generalized Lax pairs and the extended classical Yang-Baxter equation.

Inspired by these works, we consider the Kupershmidt-(dual-)Nijenhuis structure on Malcev algebras, characterize the relationships between Nijenhuis operators and Kupershmidt operators and study deformations of Kupershmidt operators.

The paper is organized as follows. First, we recall some basic definitions about alternative and Malcev algebras. In Section 2, we study infinitesimal deformations of a Malcev algebra with a representation, introduce the notion of Nijenhuis pair on a Malcev algebra and show that it generates a trivial deformation of a Lie algebra with a representation. We also introduce the notion of dual-Nijenhuis pair as the dual of a Nijenhuis pair. In Section 3, we introduce the notions of Kupershmidt-Nijenhuis structure and Kupershmidtdual-Nijenhuis structure. Some properties of Kupershmidt-(dual-)Nijenhuis structures are studied. In Section 4, we first give the relations between Nijenhuis operators and Kupershmidt operators. Then, we prove that, on the one hand, Kupershmidt-(dual-)Nijenhuis structures give rise to hierarchies of Kupershmidt operators, which are pairwise compatible; on the other hand, compatible Kupershmidt operators with a condition can give a Kupershmidt-dual-Nijenhuis structure. In Section 5, we study deformations of Kuperchmidt operators and introduce the notion of Nijenhuis elements.

Throughout this paper $\mathbb{K}$ is a field of characteristic 0 and all vector spaces are over $\mathbb{K}$.

\section{Definitions and preliminary results}

The purpose of this section is to recall the notions of alternative and Malcev algebras and their general properties (see [23] for more details).

Definition 2.1. An alternative algebra $(A, \circ)$ is a vector space $A$ equipped with a bilinear operation $(x, y) \rightarrow x \circ y$ satisfying the following condition, for all $x, y, z \in A$

$$
a s(x, x, y)=a s(y, x, x)=0,
$$


where $a s(x, y, z)=(x \circ y) \circ z-x \circ(y \circ z)$ is the associator.

Definition 2.2. [23] A Malcev algebra is a vector space $A$ endowed with an skewsymmetric bilinear product $[$,$] satisfying the Malcev identity$

$$
J(x, y,[x, z])=[J(x, y, z), x],
$$

for all $x, y, z \in A$, where $J(x, y, z)=[[x, y], z]+[[y, z], x]+[[z, x], y]$ is the Jacobiator of $x, y, z$.

Remark 2.3. [30] The Malcev identity is equivalent to Sagle's identity:

$$
[[x, z],[y, t]]=[[[x, y], z], t]+[[[y, z], t], x]+[[[z, t], x], y]+[[[t, x], y], z]
$$

for all $x, y, z, t \in A$.

Let $(A,[]$,$) and \left(A^{\prime},[,]^{\prime}\right)$ be two Malcev algebras. A linear map $f: A \rightarrow A^{\prime}$ is said to be a morphism of Malcev algebras if

$$
[f(x), f(y)]^{\prime}=f([x, y]) \text {, for all } x, y \in A .
$$

Theorem 2.4. The alternative algebra is a Malcev-admissible algebra. That is, if $(A, \circ)$ is an alternative algebra, then $(A,[]$,$) is a Malcev algebra, where$

$$
[x, y]=x \circ y-y \circ x, \forall x, y \in A \text {. }
$$

Definition 2.5. [20] A representation of a Malcev algebra $(A,[]$,$) on a vector space V$ is a map $\varrho: M \longrightarrow \operatorname{End}(V)$ such that

$$
\varrho([[x, y], z])=\varrho(x) \varrho(y) \varrho(z)-\varrho(z) \varrho(x) \varrho(y)+\varrho(y) \varrho([z, x])-\varrho([y, z]) \varrho(x)
$$

for all $x, y, z \in A$.

Example 2.6. Let $(A,[]$,$) be a Malcev algebra. Then a d_{x}: A \longrightarrow \operatorname{End}(A)$ defined by

$$
a d_{x}(y)=[x, y], \quad \forall x, y \in A,
$$

is a representation of $(A,[]$,$) on A$, which is called the adjoint representation of $\mathrm{A}$.

Proposition 2.7. Let $(A,[]$,$) be a Malcev algebra, V$ a vector space and $\varrho: A \longrightarrow$ $\operatorname{End}(V)$ a linear map. Then $(V, \varrho)$ is a representation of $A$ if and only if $(A \oplus V,[,] \varrho)$ is a Malcev algebra, where $[,]_{\varrho}$ is defined by

$$
[x+a, y+b]_{\varrho}=[x, y]+\varrho(x) a-\varrho(y) b
$$

for all $x, y \in A, a, b \in V$. This Malcev algebra is called the semidirect product of $(A,[]$, and $V$ and denoted by $A \ltimes_{\varrho} V$.

Let $(A,[]$,$) be a Malcev algebra and (V, \varrho)$ is a representation on $A$. Define the linear $\operatorname{map} \varrho^{*}: A \longrightarrow A l\left(V^{*}\right)$ by

$$
\left\langle\varrho^{*}(x)(\alpha), v\right\rangle=-\langle\alpha, \varrho(x)(v)\rangle, \quad \forall x, y \in A, v \in V, \alpha \in V^{*} .
$$

Proposition 2.8. With the above notation, $\left(V^{*}, \varrho^{*}\right)$ is a representation on $A$.

Corollary 2.9. Let $(A,[]$,$) be a Malcev algebra and (V, \varrho)$ is a representation on $A$. Then, there exist a Malcev structure on $A \oplus V^{*}$ denoted by $A \ltimes \varrho_{\varrho^{*}} V^{*}$ and given by

$$
[x+a, y+b]_{A \oplus V^{*}}=[x, y]+\varrho^{*}(x) \xi-\varrho^{*}(y) \zeta
$$

for all $x, y \in A, \xi, \zeta \in V^{*}$.

Now, we introduce the notion of Kupershmidt operator (also called an O-operator).

Definition 2.10. A Kupershmidt operator on a Malcev algebra $(A,[$,$] with respect to$ a representation $(V ; \varrho)$ is a linear map $T: V \longrightarrow A$ satisfying

$$
[T(u), T(v)]=T(\varrho(T(u))(v)-\varrho(T(v))(u)), \quad \forall u, v \in V .
$$

Example 2.11. A Rota-Baxter operator $R: A \rightarrow A$ is a Kupershmidt operator on a Malcev algebra $(A,[]$,$) with respect to the adjoint representation (A ; \mathrm{ad})$. 


\section{Infinitesimal deformations of a Malcev algebra with a representation}

Let $(A,[]$,$) be a Malcev algebra and \varrho: A \longrightarrow \operatorname{End}(V)$ be a representation. Let $\omega: \wedge^{2} A \longrightarrow A$ and $\theta: A \longrightarrow \operatorname{End}(V)$ be linear maps. Consider a $t$-parametrized family of bracket operations and linear maps:

$$
\begin{aligned}
{[x, y]_{t} } & =[x, y]+t \omega(x, y), \\
\varrho_{t}(x) & =\varrho(x)+t \theta(x) .
\end{aligned}
$$

If $\left(A,[,]_{t}\right)$ are Malcev algebras and $\varrho_{t}$ are representations of $\left(A,[,]_{t}\right)$ on $V$ for all $t$, we say that $(\omega, \theta)$ generates a one-parameter infinitesimal deformation of the Malcev algebra $(A,[]$,$) with the representation (V ; \varrho)$. We denote a one-parameter infinitesimal deformation of a Malcev algebra $(A,[]$,$) with a representation (V ; \varrho)$ by $\left(A,[,]_{t}, \varrho_{t}\right)$.

By direct calculation, we can deduce that $\left(A,[,]_{t}, \varrho_{t}\right)$ is a one-parameter infinitesimal deformation of the Malcev algebra $(A,[]$,$) with the representation (V ; \varrho)$ if and only if

$$
\begin{aligned}
& \omega([x, z],[y, t])-\omega([[x, y], z], t)-\omega([[y, z], t], x)-\omega([[z, t], x], y)+\omega([[t, x], y], z) \\
& +[\omega(x, z),[y, t]]-[\omega([x, y], z), t]-[\omega([y, z], t), x]-[\omega([z, t], x), y]-[\omega([t, x], y), z] \\
& +[[x, z], \omega(y, t)]-[[\omega(x, y), z], t]-[[\omega(y, z), t], x]-[[\omega(z, t), x], y]-[[\omega(t, x), y], z]=0, \\
& \omega(\omega(x, z),[y, t])-\omega([\omega(x, y), z], t)-\omega([\omega(y, z), t], x)-\omega(\omega([z, t], x), y)+\omega(\omega([t, x], y), z) \\
& +\omega([x, z], \omega(y, t))-\omega([\omega(x, y), z], t)-\omega([\omega(y, z), t], x)-\omega([\omega(z, t), x], y)+\omega([\omega(t, x), y], z) \\
& +[\omega(x, z), \omega(y, t)]-[\omega(\omega(x, y), z), t]-[\omega(\omega(y, z), t), x]-[\omega(\omega(z, t), x), y]-[\omega(\omega(t, x), y), z] \\
& =0,
\end{aligned}
$$

$$
\begin{aligned}
& \omega(\omega(x, z), \omega(y, t))-\omega(\omega(\omega(x, y), z), t)-\omega(\omega(\omega(y, z), t), x) \\
& -\omega(\omega(\omega(z, t), x), y)-\omega(\omega(\omega(t, x), y), z)=0,
\end{aligned}
$$

$$
\begin{aligned}
& \theta([[x, y], z])-\theta(x) \varrho(y) \varrho(z)+\theta(z) \varrho(x) \varrho(y)-\theta(y) \varrho([z, x])+\theta([y, z]) \varrho(x) \\
& +\varrho(\omega([x, y], z))-\varrho(x) \theta(y) \varrho(z)+\varrho(z) \theta(x) \varrho(y)-\varrho(y) \theta([z, x])+\varrho(\omega(y, z)) \varrho(x) \\
& \varrho([\omega(x, y), z])-\varrho(x) \varrho(y) \theta(z)+\varrho(z) \varrho(x) \theta(y)-\varrho(y) \varrho(\omega(z, x))+\varrho([y, z]) \theta(x)=0, \\
& \theta(\omega([x, y], z))-\theta(x) \theta(y) \varrho(z)+\theta(z) \theta(x) \varrho(y)-\theta(y) \theta([z, x])+\theta(\omega(y, z)) \varrho(x) \\
& +\theta([\omega(x, y), z])-\theta(x) \varrho(y) \theta(z)+\theta(z) \varrho(x) \theta(y)-\theta(y) \varrho(\omega(z, x))+\theta([y, z]) \theta(x) \\
& +\varrho(\omega(\omega(x, y), z))-\varrho(x) \theta(y) \theta(z)+\varrho(z) \theta(x) \theta(y)-\varrho(y) \theta(\omega(z, x))+\varrho(\omega(y, z)) \theta(x)=0, \\
& \theta(\omega(\omega(x, y), z))-\theta(x) \theta(y) \theta(z)+\theta(z) \theta(x) \theta(y)-\theta(y) \theta(\omega(z, x))+\theta(\omega(y, z)) \theta(x)=0 .
\end{aligned}
$$

It is well-known that (6.3) means that $\omega$ is a 2-cocycle of the Malcev algebra $A$ with the coefficient in the adjoint representation and (3.3) means that $(A, \omega)$ is a Malcev algebra. Furthermore, (3.6) means that $\theta$ is a representation of the Malcev algebra $(A, \omega)$ on $V$ and (3.4)-(3.5) means that $\varrho+\theta$ is a representation of the Malcev algebra $(A,[]+,\omega()$,$) on$ $V$.

Definition 3.1. Two one-parameter infinitesimal deformations, $\left(A,[,]_{t}, \varrho_{t}\right)$ and $\left(A,[,]_{t}^{\prime}, \varrho_{t}^{\prime}\right)$, of a Malcev algebra $(A,[]$,$) with a representation (V ; \varrho)$ are equivalent if there exists an isomorphism $\left(\operatorname{Id}+t N, \operatorname{Id}_{V}+t S\right)$ from $\left(A,[,]_{t}^{\prime}, \varrho_{t}^{\prime}\right)$ to $\left(A,[,]_{t}, \varrho_{t}\right)$, i.e.

$$
\begin{aligned}
(\operatorname{Id}+t N)[x, y]_{t}^{\prime} & =[(\operatorname{Id}+t N)(x),(\operatorname{Id}+t N)(y)]_{t}, \\
\left(\operatorname{Id}_{V}+t S\right) \varrho_{t}^{\prime}(x) & =\varrho_{t}((\operatorname{Id}+t N)(x))\left(\operatorname{Id}_{V}+t S\right) .
\end{aligned}
$$

A one-parameter infinitesimal deformation of a Malcev algebra $(A,[]$,$) with a repre-$ sentation $(V ; \varrho)$ is said to be trivial if it is equivalent to $(A,[],, \varrho)$. 
The deformation $\left(A,[,]_{t}, \varrho_{t}\right)$ is trivial if and only if

$$
\begin{aligned}
\omega(x, y) & =[N(x), y]+[x, N(y)]-N([x, y]), \\
N \omega(x, y) & =[N(x), N(y)], \\
\theta(x) & =\varrho(N(x))+\varrho(x) S-S \varrho(x), \\
\varrho(N(x)) S & =S \theta(x)
\end{aligned}
$$

for all $x, y \in A$. Using (3.7) and (3.8) that $N$ must be a Nijenhuis operator on the Malcev algebra $(A,[]$,$) . More precisely, A Nijenhuis operator on a Malcev algebra (A,[]$, is a linear map $N: A \longrightarrow A$ satisfying

$$
[N(x), N(y)]=N([N(x), y]+[x, N(y)]-N[x, y]), \quad \forall x, y \in A .
$$

Furthermore, define the bracket $[,]_{N}: \wedge^{2} A \longrightarrow A$ given by

$$
[x, y]_{N}=[N(x), y]+[x, N(y)]-N([x, y]) .
$$

Proposition 3.2. With the above notation, $\left(A,[,]_{N}\right)$ is a Malcev algebra and $N$ is a Malcev algebra morphism from $\left(A,[,]_{N}\right)$ to $(A,[]$,$) .$

It follows from (3.9) and (3.10) that $N$ and $S$ should satisfy the condition:

$$
\varrho(N(x))(S(v))=S(\varrho(N x)(v))+S(\varrho(x) S(v))-S^{2}(\varrho(x)(v)), \forall x \in A, v \in V .
$$

Definition 3.3. A pair $(N, S)$, where $N \in \operatorname{End}(A)$ and $S \in \operatorname{End}(V)$, is called a Nijenhuis pair on a Malcev algebra $(A,[]$,$) with a representation (V ; \varrho)$ if $N$ is a Nijenhuis operator on the Malcev algebra $(A,[]$,$) and the condition (3.13)$ holds.

We have seen that a trivial deformation of a Malcev algebra with a representation could give rise to a Nijenhuis pair. In fact, the converse is also true.

Theorem 3.4. Let $(N, S)$ be a Nijenhuis pair on a Malcev algebra $(A,[]$,$) with a$ representation $(V ; \varrho)$. Then a deformation of $(A,[],, \varrho)$ can be obtained by putting

$$
\begin{aligned}
\omega(x, y) & =[N(x), y]+[x, N(y)]-N([x, y]) ; \\
\theta(x) & =\varrho(N(x))+\varrho(x) S-S \varrho(x) .
\end{aligned}
$$

Furthermore, this deformation is trivial.

Proof. It is a straightforward computations. We omit the details.

Now, we introduce the notion of a dual-Nijenhuis pair on a Malcev algebra with a representation.

Definition 3.5. A pair $(N, S)$, where $N \in \operatorname{End}(A)$ and $S \in \operatorname{End}(V)$, is called a dualNijenhuis pair on a Malcev algebra $(A,[]$,$) with a representation (V ; \varrho)$ if $N$ is a Nijenhuis operator on the Malcev algebra $(A,[]$,$) and S$ satisfies the following condition:

$$
\varrho(N(x))(S(v))=S(\varrho(N(x))(v))+\varrho(x)\left(S^{2}(v)\right)-S(\varrho(x)(S(v))) .
$$

In fact, there is a close relationship between a Nijenhuis pair and a dual-Nijenhuis pair.

Proposition 3.6. Let $(A,[]$,$) be a Malcev algebra. Then, (N, S)$ is a Nijenhuis pair on $A$ with a representation $(V ; \varrho)$ if and only if $\left(N, S^{*}\right)$ is a dual-Nijenhuis pair on $A$ with the representation $\left(V^{*} ; \varrho^{*}\right)$.

Proof. For any $x \in A, v \in V$ and $\xi \in V^{*}$, we have

$$
\begin{aligned}
& \left\langle\varrho(N(x)) S(v)-S \varrho(N(x))(v)-S \varrho(x) S(v)+S^{2} \varrho(x)(v), \xi\right\rangle \\
= & \langle\varrho(N(x)) S(v), \xi\rangle-\langle S \varrho(N(x))(v), \xi\rangle-\langle S \varrho(x) S(v), \xi\rangle+\left\langle S^{2} \varrho(x)(v), \xi\right\rangle \\
= & -\left\langle v, S^{*} \varrho^{*}(N(x))(\xi)\right\rangle+\left\langle v, \varrho^{*}(N(x))\left(S^{*}(\xi)\right)\right\rangle+\left\langle v, S^{*} \varrho^{*}(x)\left(S^{*}(\xi)\right)\right\rangle-\left\langle v, \varrho^{*}(x)\left(\left(S^{*}\right)^{2}(\xi)\right)\right\rangle \\
= & -\left\langle v, S^{*} \varrho^{*}(N(x))(\xi)-\varrho^{*}(N(x))\left(S^{*}(\xi)\right)-S^{*} \varrho^{*}(x)\left(S^{*}(\xi)\right)+\varrho^{*}(x)\left(\left(S^{*}\right)^{2}(\xi)\right)\right\rangle .
\end{aligned}
$$


Definition 3.7. A Nijenhuis pair $(N, S)$ on a Malcev algebra $(A,[]$,$) with a representa-$ tion $(V ; \varrho)$ is called a perfect Nijenhuis pair if

$$
S^{2}(\varrho(x)(v))+\varrho(x)\left(S^{2}(v)\right)=2 S(\varrho(x)(S(v))), \quad \forall x \in A, v \in V .
$$

It is obvious that a perfect Nijenhuis pair is not only a Nijenhuis pair but also a dualNijenhuis pair. A Nijenhuis pair gives rise to a Nijenhuis operator on the semidirect product Malcev algebra.

Proposition 3.8. Let $(N, S)$ be a Nijenhuis pair on a Malcev algebra $(A,[]$,$) with a$ representation $(V ; \varrho)$. Then $N+S$ is a Nijenhuis operator on the semidirect product Malcev algebra $A \ltimes_{\varrho} V$. Furthermore, if $(N, S)$ is a perfect Nijenhuis pair, then $N+S^{*}$ is a also a Nijenhuis operator on the semidirect product Malcev algebra $A \ltimes_{\varrho^{*}} V^{*}$.

Proof. Let $x, y \in A$ and $u, v \in V$. Using Eqs (3.11) and (3.13), we have

$$
\begin{aligned}
& {[(N+S)(x+u),(N+S)(y+v)]_{\varrho}-(N+S)\left([(N+S)(x+u), y+v]_{\varrho}\right.} \\
& \left.+[x+u,(N+S)(y+v)]_{\varrho}-(N+S)([x+u, y+v]) \varrho\right) \\
= & {[(N(x), N(y)]-[N(x), y]-[x, N(y)]+N([x, y])+\varrho(N(x))(S(v))-S(\varrho(N x)(v))} \\
& -S(\varrho(x) S(v))+S^{2}(\varrho(x)(v))+\varrho(N(y))(S(u))-S(\varrho(N y)(u))-S(\varrho(y) S(u)) \\
& +S^{2}(\varrho(y)(u)) \\
= & 0 .
\end{aligned}
$$

Similarly, we can check that, if $(N, S)$ is a perfect Nijenhuis pair, then $N+S^{*}$ is a Nijenhuis operator on $A \ltimes_{\varrho^{*}} V^{*}$.

We have the following straightforward proposition.

Proposition 3.9. Let $(N, S)$ be a Nijenhuis operator on an alternative algebra $(A, \circ)$. Then $N$ is a Nijenhuis operator on the Malcev algebra given in Theorem 2.4.

Now, let $(A,[]$,$) be a Malcev algebra. Define \hat{\varrho}: A \longrightarrow \operatorname{End}(V)$ and $\tilde{\varrho}: A \longrightarrow \operatorname{End}(V)$, respectively, by

$$
\begin{aligned}
& \hat{\varrho}(x)=\varrho(N x)+[\varrho(x), S], \\
& \tilde{\varrho}(x)=\varrho(N x)-[\varrho(x), S], \quad \forall x \in A .
\end{aligned}
$$




\section{Proposition 3.10.}

(1) If $(N, S)$ is a Nijenhuis pair on $A$ with a representation $\varrho$, then $\varrho$ is a representation of the Malcev algebra $\left(A,[,]_{N}\right)$ on $V$;

(2) If $(N, S)$ is a dual-Nijenhuis pair on $A$ with a representation $\varrho$, then $\varrho$ is a representation of the Malcev algebra $\left(A,[,]_{N}\right)$ on $V$;

Proof. Item 1. By Proposition 3.8, $N+S$ is a Nijenhuis operator on the semidirect product Malcev algebra $A \ltimes_{\varrho} V$. The deformed bracket $[,]_{N+S}$ is given by

$$
\begin{aligned}
{[x+u, y+v]_{N+S}=} & {[(N+S)(x+u), y+v]_{\varrho}+[x+u,(N+S)(y+v)]_{\varrho} } \\
& -(N+S)[x+u, y+v]_{\varrho} \\
= & {[N x, y]+[x, N y]-N[x, y] } \\
& +\varrho(N x)(v)+\varrho(x) S(v)-S \varrho(x)(v)-\varrho(y) S(u)-\varrho(N y)(u) \\
& +S \varrho(y)(u) \\
= & {[x, y]_{N}+\varrho(x)(v)-\varrho(y)(u), }
\end{aligned}
$$

which implies that $\hat{\varrho}$ is a representation of the Malcev algebra $\left(A,[,]_{N}\right)$ on $V$.

Item 2. By direct calculation, the dual map $\tilde{\varrho}^{*}$ of $\tilde{\varrho}$ is given by

$$
\tilde{\varrho}^{*}(x)=\varrho^{*}(N x)+\left[\varrho^{*}(x), S^{*}\right], \quad \forall x \in A .
$$

Since $(N, S)$ is a dual-Nijenhuis pair with a representation $\varrho$, by Proposition $3.6,\left(N, S^{*}\right)$ is a Nijenhuis pair with a representation $\varrho^{*}$. By $(1), \varrho^{*}$ is a representation of the Malcev algebra $\left(A,[,]_{N}\right)$ on $V^{*}$ and thus $\varrho$ is a representation of the Lie algebra $\left(A,[,]_{N}\right)$ on $V$.

\section{Kupershmidt-(dual-)Nijenhuis structures}

There is a close relationship between Kupershmidt operators and pre-Malcev algebras.

Definition 4.1. [22] A pre-Malcev algebra is a vector space A endowed with a bilinear product ". " $A \times A \rightarrow A$ satisfying the identity

$$
([y, z]) \cdot(x \cdot t)+([[x, y], z]) \cdot t+y \cdot(([x, z]) \cdot t)-x \cdot(y \cdot(z \cdot t))+z \cdot(x \cdot(y \cdot t))=0
$$

for all $x, y, z, t \in A$, where $[x, y]=x \cdot y-y \cdot x$.

The identity $(4.2)$ is equivalent to

$$
\begin{aligned}
0 & =(y \cdot z) \cdot(x \cdot t)-(z \cdot y) \cdot(x \cdot t) \\
& +((x \cdot y) \cdot z) \cdot t-((y \cdot x) \cdot z) \cdot t-(z \cdot(x \cdot y)) \cdot t+(z \cdot(y \cdot x)) \cdot t \\
& +y \cdot((x \cdot z) \cdot t)-y \cdot((z \cdot x) \cdot t)-x \cdot(y \cdot(z \cdot t))+z \cdot(x \cdot(y \cdot t))
\end{aligned}
$$

for all $x, y, z, t \in A$.

Proposition 4.2. Let $(A, \cdot)$ be a pre-Malcev algebra. Then The commutator $[x, y]=$ $x \cdot y-y \cdot x$ defines a Malcev algebra $A$. which is called the sub-adjacent Malcev algebra of $(A, \cdot)$ and denoted by $A^{C}$. Furthermore, $L: A \longrightarrow A l(A)$ with $x \rightarrow L_{x}$, where $L_{x} y=x \cdot y$, for all $x, y \in A$, gives a representation of the Malcev algebra $A^{C}$ on $A$.

The following result establishes the connection between Kupershmidt operators and pre-Malcev algebras which generalize the construction with Rota-Baxter operators (see in $[22]$ for more details). A Kupershmidt operator on a Malcev algebra $(A,[]$,$) with respect$ to a representation $(V ; \varrho)$ is a linear map $T: V \rightarrow A$ satisfying

$$
[T(u), T(v)]=T(\varrho(T(u))(v)-\varrho(T(v))(u)), \forall u, v \in V .
$$


Theorem 4.3. Let $T: V \rightarrow A$ be a Kupershmidt operator on a Malcev algebra $(A,[]$, with respect to a representation $(V ; \varrho)$. Define a multiplication $\cdot$ on $V$ by

$$
u \cdot{ }^{T} v=\varrho(T u)(v), \quad \forall u, v \in V .
$$

Then $\left(V,{ }^{T}\right)$ is a pre-Malcev algebra.

Proof. Using the identity of Kupershmidt operator (2.6), we have

$$
T\left([u, v]^{T}\right)=T\left(u \cdot \cdot^{T} v-v \cdot{ }^{T} u\right)=[T(u), T(v)] .
$$

Thanks to Eq. (2.4), for $a, b, u, v, \in V$, we get

$$
\begin{aligned}
& \left(\left[[a, b]^{T}, v\right]^{T}\right) \cdot{ }^{T} t+\left([b, u]^{T}\right) \cdot{ }^{T}\left(a \cdot{ }^{T} v\right)+b \cdot{ }^{T}\left(\left([a, u]^{T}\right) \cdot{ }^{T} v\right)-a \cdot{ }^{T}\left(b \cdot{ }^{T}\left(u \cdot{ }^{T} v\right)\right) \\
& +u \cdot \cdot^{T}\left(a \cdot{ }^{T}\left(b \cdot{ }^{T} v\right)\right) \\
= & \varrho\left(T\left(\left[[a, b]^{T}, u\right]^{T}\right)\right) v-\varrho(T(u)) \varrho(T(b)) \varrho(T(u)) v \\
& +\varrho(T(u)) \varrho(T(a)) \varrho(T(b)) v-\varrho(T(b)) \varrho\left(T\left([u, a]^{T}\right)\right) v+\varrho\left(T\left([b, u]^{T}\right)\right) \varrho(T(a)) v \\
= & \varrho([[T(a), T(b)], T(u)]) v-\varrho(T(u)) \varrho(T(b)) \varrho(T(u)) v \\
& +\varrho(T(u)) \varrho(T(a)) \varrho(T(b)) v-\varrho(T(b)) \varrho([T(u), T(a)]) v+\varrho([T(b), T(u)]) \varrho(T(a)) v \\
= & 0 .
\end{aligned}
$$

We denote by $\left(V,[,]^{T}\right)$ the sub-adjacent Malcev algebra of the pre-Malcev algebra $\left(V, \cdot^{T}\right)$. More precisely,

$$
[u, v]^{T}=\varrho(T u)(v)-\varrho(T v)(u)
$$

Moreover, $T$ is a Malcev algebra homomorphism from $\left(V,[,]^{T}\right)$ to $(A,[]$,$) .$

Now let $T: V \longrightarrow A$ be a Kupershmidt operator and $(N, S)$ a (dual-)Nijenhuis pair on a Malcev algebra $(A,[]$,$) with a representation (V ; \varrho)$. We define the bracket $[,]_{S}^{T}$ : $\wedge^{2} V \longrightarrow V$ to be the deformed bracket of $[,]^{T}$ by $S$, i.e.

$$
[u, v]_{S}^{T}=[S(u), v]^{T}+[u, S(v)]^{T}-S\left([u, v]^{T}\right), \quad \forall u, v \in V .
$$

Define the bracket $\{,\}_{\hat{\varrho}}^{T}: \wedge^{2} V \longrightarrow V$ and $\{,\}_{\tilde{\varrho}}^{T}: \wedge^{2} V \longrightarrow V$ similar as (4.5) using the representation $\hat{\varrho}$ and $\tilde{\varrho}$, respectively:

$$
\begin{aligned}
& \{u, v\}_{\hat{\varrho}}^{T}=\hat{\varrho}(T u)(v)-\hat{\varrho}(T v)(u), \\
& \{u, v\}_{\tilde{\varrho}}^{T}=\tilde{\varrho}(T u)(v)-\tilde{\varrho}(T v)(u), \quad \forall u, v \in V .
\end{aligned}
$$

It is not true in general that the brackets $[,]_{S}^{T},\{,\}_{\hat{\varrho}}^{T}$ and $\{,\}_{\tilde{\varrho}}^{T}$ satisfy the Malcev identity.

Definition 4.4. A Kupershmidt operator $T: V \longrightarrow A$ on a Malcev algebra $(A,[]$,$) with$ respect to a representation $(V ; \varrho)$ and a (dual-)Nijenhuis pair $(N, S)$ are called compatible if they satisfy the following conditions

$$
\begin{aligned}
N T & =T S, \\
{[u, v]^{N T} } & =[u, v]_{S}^{T}
\end{aligned}
$$

for all $u, v \in V$. The triple $(T, S, N)$ is called a Kupershmidt-(dual-)Nijenhuis structure on the Malcev algebra $(A,[]$,$) with respect to the representation (V ; \varrho)$ if $T$ and $(N, S)$ are compatible.

Note that if $(N, S)$ is a perfect Nijenhuis pair on a Malcev algebra $(A,[]$,$) , then a$ Kupershmidt-Nijenhuis structure on $A$ is also a Kupershmidt-dual-Nijenhuis structure on $A$. 
Lemma 4.5. Let $(T, S, N)$ be a Kupershmidt-Nijenhuis structure on a Malcev algebra $(A,[]$,$) with respect to the representation (V ; \varrho)$. Then we have

for all $u, v \in V$.

$$
[u, v]_{S}^{T}=\{u, v\}_{\hat{\varrho}}^{T} \text { and }[u, v]_{S}^{T}=\{u, v\}_{\tilde{\varrho}}^{T}
$$

Proof. It easy to check, from (4.8) and for all $u, v \in V,[u, v]_{S}^{T}=\{u, v\}_{\hat{\varrho}}^{T}$.

By (4.8), we have

$$
[u, v]_{S}^{T}+\{u, v\}_{\tilde{\varrho}}^{T}=2[u, v]^{N T} .
$$

Then by (4.9), we obtain $[u, v]_{S}^{T}=\{u, v\}_{\tilde{\varrho}}^{T}$.

Thus, if $(T, S, N)$ is a Kupershmidt-(dual-)Nijenhuis structure, then the three brackets $[,]_{S}^{T},\{,\}_{\hat{\varrho}}^{T}$ and $[,]^{N T}$ are the same. Moreover, we will see that they satisfy the Jacobi identity.

Proposition 4.6. Let $(T, S, N)$ be a Kupershmidt-(dual-)Nijenhuis structure on a Malcev algebra $(A,[]$,$) with respect to the representation (V ; \varrho)$. Then $S$ is a Nijenhuis operator on the sub-adjacent Malcev algebra $\left(V,[,]^{T}\right)$. Thus, the brackets $[,]_{S}^{T},\{,\}_{\hat{\varrho}}^{T}\left(\{,\}_{\tilde{\varrho}}^{T}\right)$ and $[,]^{N T}$ are all Malcev brackets.

Proof. For the Kupershmidt-Nijenhuis structure $(T, S, N)$, by (3.13) and substituting $x$ by $T(u)$, we get

$$
\begin{aligned}
0 & =\varrho(N T(u)) S(v)-S(\varrho(N T(u))(v)+S \varrho(T(u)) S(v)-S \varrho(T(u))(v)) \\
& =\varrho(T S(u)) S(v)-S(\varrho(T S(u))(v)+S \varrho(T u) S(v)-S \varrho(T u)(v)) \\
& =S(u) \cdot S(v)-S(S(u) \cdot v+u \cdot S(v)-S(u \cdot v)),
\end{aligned}
$$

which implies that $S$ is a Nijenhuis operator on the pre-Malcev algebra $(V, \cdot)([22])$. Thus $S$ is a Nijenhuis operator on the sub-adjacent Malcev algebra $\left(V,[,]^{T}\right)$.

For the Kupershmidt-dual-Nijenhuis structure $(T, S, N)$, the proof is not direct. In fact, by the relation $[u, v]^{T S}=[u, v]_{S}^{T}$, one has

$$
\begin{aligned}
S(\varrho(T S(u))(v))-S(\varrho(T(v))(S(u))) & =\varrho(T S(u))(S(v))-\varrho(T(v))\left(S^{2}(u)\right) ; \\
S^{2}(\varrho(T(u))(v))-S^{2}(\varrho(T(v))(u)) & =S(\varrho(T(u))(S(v)))-S(\varrho(T(v))(S(u)))
\end{aligned}
$$

By the condition (3.16), we have

$$
\varrho(T(v))\left(S^{2}(u)\right)-\varrho(T S(v))(S(u))=S(\varrho(T(v))(S(u)))-S(\varrho(T S(v))(u)) .
$$

By (4.10)-(4.12), we have

$$
\begin{aligned}
& {[S(u), S(v)]^{T}-S\left([u, v]_{S}^{T}\right) } \\
= & \varrho(T S(u))(S(v))-\varrho(T S(v))(S(u))+S^{2}(\varrho(T(u))(v))-S^{2}(\varrho(T(v))(u)) \\
& -S(\varrho(T(u))(S(v)))+S(\varrho(T S(v))(u))-S(\varrho(T S(u))(v))+S(\varrho(T(v))(S(u))) \\
= & \varrho(T S(u))(S(v))-\varrho(T S(v))(S(u))-S^{2}(\varrho(T(v))(u))+S^{2}(\varrho(T(u))(v)) \\
& -S(\varrho(T(u))(S(v)))+S(\varrho(T S(v))(u))-\varrho(T S(u))(S(v))+S\left(\varrho(T(v))\left(S^{2}(u)\right)\right) \\
= & \varrho(T S(u))(S(v))-\varrho(T S(v))(S(u))+S(\varrho(T(u))(S(v)))-\varrho(T(v))(S(u)) \\
& -S(\varrho(T(u))(S(v)))+S(\varrho(T S(v))(u))-\varrho(T S(u))(S(v))+\varrho(T(v))\left(S^{2}(u)\right) \\
= & \varrho(T(v))\left(S^{2}(u)\right)-\varrho(T S(v))(S(u))-S(\varrho(T(v))(S(u)))+S(\varrho(T S(v))(u)) \\
= & 0 .
\end{aligned}
$$

Thus $S$ is a Nijenhuis operator on the Malcev algebra $\left(V,[,]^{T}\right)$.

Theorem 4.7. Let $(T, S, N)$ be a Kupershmidt-(dual-)Nijenhuis structure on a Malcev algebra $(A,[]$,$) with respect to the representation (V ; \varrho)$. Then we have 
(1) $T$ is a Kupershmidt operator on the deformed Malcev algebra $\left(A,[,]_{N}\right)$ with respect to the representation $(V ; \varrho)((V ; \varrho))$;

(2) NT is a Kupershmidt operator on the Malcev algebra $\left(A,[,]_{A}\right)$ with respect to the representation $(V ; \varrho)$.

Proof. We only prove the theorem for the Kupershmidt-Nijenhuis structure. The other one can be proved similarly.

Item 1 . Since $T$ is a Kupershmidt operator on the Malcev algebra $(A,[]$,$) with respect$ to the representation $(V ; \varrho)$ and $T S=N T$, we have, for $u, v \in V$

$$
\begin{aligned}
T\{u, v\}_{\hat{\varrho}}^{T} & =T\left([u, v]_{S}^{T}\right)=T\left([S(u), v]^{T}+[u, S(v)]^{T}-S[u, v]^{T}\right) \\
& =[T S(u), T(v)]+[T(u), T S(v)]-T S[u, v]^{T} \\
& =[N T(u), T(v)]+[T(u), N T(v)]-N T[u, v]^{T} \\
& =[T(u), T(v)]_{N} .
\end{aligned}
$$

Thus, $T$ is a Kupershmidt operator on the deformed Malcev algebra $\left(A,[,]_{N}\right)$ with respect to the representation $(V ; \hat{\varrho})$.

Item 2. By (4.9), we have

$$
N T\left([u, v]^{N T}\right)=N T\left([u, v]_{S}^{T}\right)=N[T(u), T(v)]_{N}=[N T(u), N T(v)],
$$

which implies that $N T$ is a Kupershmidt operator on the Malcev algebra $(A,[]$,$) with$ respect to the representation $(V ; \varrho)$.

The following theorem demonstrates that the Kupershmidt-Nijenhuis operator can give a Kupershmidt-dual-Nijenhuis operator with a condition.

Theorem 4.8. Let $(T, S, N)$ be a Kupershmidt-Nijenhuis structure on a Malcev algebra $(A,[]$,$) with respect to the representation (V ; \varrho)$. If $T$ is invertible, then $(T, S, N)$ is a Kupershmidt-dual-Nijenhuis structure.

Proof. We only need to prove that the Nijenhuis pair $(S, N)$ is also a dual-Nijenhuis pair. By (4.9), we have

$$
[u, v]_{S}^{T}-[u, v]^{T S}=\varrho(T(u))(S(v))-\varrho(T(v))(S(u))-S(\varrho(T(u))(v)-\varrho(T(v))(u)),
$$

which impMalcevs that

$$
\varrho(T(u))(S(v))-\varrho(T(v))(S(u))=S(\varrho(T(u))(v)-\varrho(T(v))(u)) .
$$

Since $S$ is a Nijenhuis operator on the Malcev algebra $\left(V,[,]^{T}\right)$ and $[u, v]_{S}^{T}=[u, v]^{T S}$, we have

which means that

$$
S\left([u, v]^{T S}\right)=[S(u), S(v)]^{T},
$$

$$
S(\varrho(T S(u))(v)-\varrho(T S(v))(u))=\varrho(T S(u))(S(v))-\varrho(T S(v))(S(u)) .
$$

By (4.13), we have

$$
S(\varrho(T S(u))(v))-\varrho(T S(u))(S(v))=S(\varrho(T(v))(S(u)))-\varrho(T(v))\left(S^{2}(u)\right) .
$$

Thus (4.14) implies that

$$
\begin{aligned}
0 & =S(\varrho(T(v))(S(u)))-\varrho(T(v))\left(S^{2}(u)\right)-S(\varrho(T S(v))(u))+\varrho(T S(v))(S(u)) \\
& =S(\varrho(T(v))(S(u)))-\varrho(T(v))\left(S^{2}(u)\right)-S(\varrho(N T(v))(u))+\varrho(N T(v))(S(u)) .
\end{aligned}
$$

Since $T$ is invertible and let $x=T(v)$, we have

$$
S(\varrho(x)(S(u)))-\varrho(x)\left(S^{2}(u)\right)-S(\varrho(N(x))(u))+\varrho(N(x))(S(u))=0 .
$$


Thus the Nijenhuis pair $(S, N)$ is a dual-Nijenhuis pair. We finish the proof.

\section{Hierarchy of Kupershmidt operators}

\subsection{Compatible Kupershmidt operators on Malcev algebras}

Definition 5.1. Let $T_{1}, T_{2}: V \longrightarrow A$ be two Kupershmidt operators on a Malcev algebra $(A,[]$,$) with respect to a representation (V ; \varrho)$. If for all $k_{1}, k_{2} \in \mathbb{K}, k_{1} T_{1}+k_{2} T_{2}$ is still a Kupershmidt operator on $A$, then $T_{1}$ and $T_{2}$ are called compatible.

Proposition 5.2. Let $T_{1}, T_{2}: V \longrightarrow A$ be two Kupershmidt operators on a Malcev algebra $(A,[]$,$) with respect to a representation (V ; \varrho)$. Then $T_{1}$ and $T_{2}$ are compatible if and only if the following equation holds:

$$
\begin{aligned}
{\left[T_{1}(u), T_{2}(v)\right]+\left[T_{2}(u), T_{1}(v)\right]=} & T_{1}\left(\varrho\left(T_{2}(u)\right)(v)-\varrho\left(T_{2}(v)\right)(u)\right) \\
& +T_{2}\left(\varrho\left(T_{1}(u)\right)(v)-\varrho\left(T_{1}(v)\right)(u)\right), \quad \forall u, v \in V .(5.1)
\end{aligned}
$$

Proof. Let $k_{1}, k_{2} \in \mathbb{K}$ and $u, v \in A$. Then, using (4.3), we have

$$
\begin{aligned}
& {\left[\left(k_{1} T_{1}+k_{2} T_{2}\right)(u),\left(k_{1} T_{1}+k_{2} T_{2}\right)(v)\right]-\left(k_{1} T_{1}+k_{2} T_{2}\right)\left(\varrho\left(\left(k_{1} T_{1}+k_{2} T_{2}\right)(u)\right)(v)\right.} \\
& \left.+\varrho\left(\left(k_{1} T_{1}+k_{2} T_{2}\right)\right)(v)(u)\right) \\
& =k_{1}^{2}\left(\left[T_{1}(u), T_{1}(v)\right]-T_{1}\left(\varrho\left(T_{1}(u)\right)(v)+\varrho\left(T_{1}(v)\right)(u)\right)\right)+k_{2}^{2}\left(\left[T_{2}(u), T_{2}(v)\right]-T_{2}\left(\varrho\left(T_{2}(u)\right)(v)\right.\right. \\
& \left.\left.+\varrho\left(T_{2}(v)\right)(u)\right)\right)+k_{1} k_{2}\left(\left[T_{1}(u), T_{2}(v)\right]+\left[T_{2}(u), T_{1}(v)\right]-T_{1}\left(\varrho\left(T_{2}(u)\right)(v)-\varrho\left(T_{2}(v)\right)(u)\right)\right. \\
& \left.-T_{2}\left(\varrho\left(T_{1}(u)\right)(v)-\varrho\left(T_{1}(v)\right)(u)\right)\right) \\
& =k_{1} k_{2}\left(\left[T_{1}(u), T_{2}(v)\right]+\left[T_{2}(u), T_{1}(v)\right]-T_{1}\left(\varrho\left(T_{2}(u)\right)(v)-\varrho\left(T_{2}(v)\right)(u)\right)-T_{2}\left(\varrho\left(T_{1}(u)\right)(v)\right.\right. \\
& \left.\left.-\varrho\left(T_{1}(v)\right)(u)\right)\right)
\end{aligned}
$$

Thus, $T_{1}$ and $T_{2}$ are compatible if and only if the equation (5.1) holds.

Using a Kupershmidt operator and a Nijenhuis operator, we can construct a pair of compatible Kupershmidt operators .

Proposition 5.3. Let $T: V \longrightarrow A$ be a Kupershmidt operator on a Malcev algebra $(A,[]$,$) with respect to a representation (V ; \varrho)$ and $N$ a Nijenhuis operator on $(A,[]$,$) .$ Then NT is a Kupershmidt operator on the Malcev algebra $(A,[]$,$) with respect to the$ representation $(V ; \varrho)$ if and only if for all $u, v \in V$, the following equation holds:

$$
\begin{aligned}
& N([N T(u), T(v)]+[T(u), N T(v)]) \\
= & N(T(\varrho(N T(u))(v)-\varrho(N T(v))(u))+N T(\varrho(T(u))(v)-\varrho(T(v))(u))) .
\end{aligned}
$$

In this case, if in addition $N$ is invertible, then $T$ and $N T$ are compatible. More explicitly, for any Kupershmidt operator $T$, if there exists an invertible Nijenhuis operator $N$ such that NT is also a Kupershmidt operator, then $T$ and NT are compatible.

Proof. For any $u, v \in V$ and since $N$ is a Nijenhuis operator, we have

$$
[N T(u), N T(v)]=N([N T(u), T(v)]+[T(u), N T(v)])-N^{2}([T(u), T(v)]) .
$$

Then

$$
[N T(u), N T(v)]=N T(\varrho(N T(u))(v)-\varrho(N T(v))(u))
$$

if and only if (5.2) holds. 
If $N T$ is a Kupershmidt operator and $N$ is invertible, then we have

$$
\begin{aligned}
{[N T(u), T(v)]+[T(u), N T(v)]=} & T(\varrho(N T(u))(v)-\varrho(N T(v))(u))+N T(\varrho(T(u))(v) \\
& -\varrho(T(v))(u)),
\end{aligned}
$$

which is exactly the condition that $N T$ and $T$ are compatible.

A pair of compatible Kupershmidt operators can also give rise to a Nijenhuis operator under some conditions.

Proposition 5.4. Let $T_{1}, T_{2}: V \longrightarrow A$ be two Kupershmidt operators on a Malcev algebra $(A,[]$,$) with respect to a representation (V ; \varrho)$. Suppose that $T_{2}$ is invertible. If $T_{1}$ and $T_{2}$ are compatible, then $N=T_{1} T_{2}^{-1}$ is a Nijenhuis operator on the Malcev algebra $(A,[]$,$) .$

Proof. For all $x, y \in A$, there exist $u, v \in V$ such that $T_{2}(u)=x, T_{2}(v)=y$. Hence $N=T_{1} T_{2}^{-1}$ is a Nijenhuis operator if and only if the following equation holds:

$$
\left[N T_{2}(u), N T_{2}(v)\right]=N\left(\left[N T_{2}(u), T_{2}(v)\right]+\left[T_{2}(u), N T_{2}(v)\right]\right)-N^{2}\left(\left[T_{2}(u), T_{2}(v)\right]\right) .
$$

Since $T_{1}=N T_{2}$ is an Kupershmidt operator, the left hand side of the above equation is

$$
N T_{2}\left(\varrho\left(N T_{2}(u)\right)(v)-\varrho\left(N T_{2}(v)\right)(u)\right) .
$$

Since $T_{2}$ is a Kupershmidt operator which is compatible with $T_{1}=N T_{2}$, we have

$$
\begin{aligned}
& {\left[N T_{2}(u), T_{2}(v)\right]+\left[T_{2}(u), N T_{2}(v)\right] } \\
= & T_{2}\left(\varrho\left(N T_{2}(u)\right)(v)-\varrho\left(N T_{2}(v)\right)(u)\right)+N T_{2}\left(\varrho\left(T_{2}(u)\right)(v)-\varrho\left(T_{2}(v)\right)(u)\right) \\
= & T_{2}\left(\varrho\left(N T_{2}(u)\right)(v)-\varrho\left(N T_{2}(v)\right)(u)\right)+N\left(\left[T_{2}(u), T_{2}(v)\right]\right) .
\end{aligned}
$$

Let $N$ act on both sides, we get the conclusion.

By Proposition 5.3 and 5.4, we have

Corollary 5.5. Let $T_{1}, T_{2}: V \longrightarrow A$ be two Kupershmidt operators on a Malcev algebra $(A,[]$,$) with respect to a representation (V ; \varrho)$. Suppose that $T_{1}$ and $T_{2}$ are invertible. Then $T_{1}$ and $T_{2}$ are compatible if and only if $N=T_{1} T_{2}^{-1}$ is a Nijenhuis operator.

In particular, as a direct application, we have the following conclusion.

Corollary 5.6. Let $(A,[]$,$) be a Malcev algebra. Suppose that \mathcal{R}_{1}$ and $\mathcal{R}_{2}$ are two invertible Rota-Baxter operators. Then $\mathcal{R}_{1}$ and $\mathcal{R}_{2}$ are compatible in the sense that any linear combination of $\mathcal{R}_{1}$ and $\mathcal{R}_{2}$ is still a Rota-Baxter operator if and only if $N=\mathcal{R}_{1} \mathcal{R}_{2}^{-1}$ is a Nijenhuis operator.

\subsection{Hierarchy of Kupershmidt operators}

In the following, first we construct compatible Kupershmidt operators from Kupershmidt(dual-)Nijenhuis structures. Given a Kupershmidt-(dual-)Nijenhuis structure $(T, S, N)$, by Theorem 4.7, $T$ and $T S$ are Kupershmidt operators. In fact, they are compatible.

Proposition 5.7. Let $(T, S, N)$ be a Kupershmidt-(dual-)Nijenhuis structure on a Malcev algebra $(A,[]$,$) with respect to a representation (V ; \varrho)$. Then $T$ and $T S$ are compatible Kupershmidt operators.

Proof. We only prove the conclusion for the Kupershmidt-Nijenhuis structure. The other one can be proved similarly. It is sufficient to prove that $T+T S$ is a Kupershmidt operator. It is obvious that

$$
[u, v]^{T+T S}=[u, v]^{T}+[u, v]^{T S}=[u, v]^{T}+[u, v]_{S}^{T} .
$$


Thus, we have

$$
\begin{aligned}
& (T+T S)\left([u, v]^{T+T S}\right) \\
= & T\left([u, v]^{T}\right)+T S\left([u, v]_{S}^{T}\right)+T S\left([u, v]^{T}\right)+T\left([u, v]_{S}^{T}\right) \\
= & T\left([u, v]^{T}\right)+T S\left([u, v]_{S}^{T}\right)+T S\left([u, v]^{T}\right) \\
& +T\left([S(u), v]^{T}+[u, S(v)]^{T}-S[u, v]^{T}\right) \\
= & T\left([u, v]^{T}\right)+T S\left([u, v]_{S}^{T}\right)+T\left([S(u), v]^{T}+[u, S(v)]^{T}\right) \\
= & {[T(u), T(v)]+[T S(u), T S(v)]+[T S(u), T(v)]+[T(u), T S(v)] } \\
= & {[(T+T S)(u),(T+T S)(v)], }
\end{aligned}
$$

which means that $T+T S$ is a Kupershmidt operator on the Malcev algebra $(A,[]$,$) with$ respect to a representation $(V ; \varrho)$.

Lemma 5.8. Let $(T, S, N)$ be a Kupershmidt-(dual-)Nijenhuis structure on a Malcev algebra $(A,[]$,$) with respect to a representation (V ; \varrho)$. Then for all $k, i \in \mathbb{N}$, we have

$$
T_{k}[u, v]_{S^{k+i}}^{T}=\left[T_{k}(u), T_{k}(v)\right]_{N^{i}} .
$$

Proof. Since $T$ is a Kupershmidt operator and $T S=N T$, we have

$$
\begin{aligned}
T\left([u, v]_{S^{i}}^{T}\right) & =T\left(\left[S^{i}(u), v\right]^{T}+\left[u, S^{i}(v)\right]^{T}-S^{i}\left([u, v]^{T}\right)\right) \\
& =\left[N^{i}(T(u)), T(v)\right]+\left[T(u), N^{i}(T(v))\right]-N^{i}([T(u), T(v)]) \\
& =[T(u), T(v)]_{N^{i}} .
\end{aligned}
$$

Since $S$ is a Nijenhuis operator on the Malcev algebra $\left(V,[,]^{T}\right)$, we have

$$
S^{k}\left([u, v]_{S^{k+i}}^{T}\right)=\left[S^{k}(u), S^{k}(v)\right]_{S^{i}}^{T} .
$$

Then, by (5.4) and (5.5), we have

$$
T_{k}\left([u, v]_{S^{k+i}}^{T}=T S^{k}\left([u, v]_{S^{k+i}}^{T}\right)=T\left(\left[S^{k}(u), S^{k}(v)\right]_{S^{i}}^{T}\right)=\left[T\left(S^{k}(u)\right), T\left(S^{k}(v)\right)\right]_{N^{i}} .\right.
$$

The proof is finished.

Lemma 5.9. Let $(T, S, N)$ be a Kupershmidt-(dual-)Nijenhuis structure on a Malcev algebra $(A,[]$,$) with respect to a representation (V ; \varrho)$. Then for all $k, i \in \mathbb{N}$ such that $i \leq k$,

$$
[u, v]^{T_{k}}=[u, v]_{S^{k}}^{T}=S^{k-i}[u, v]^{T_{i}},
$$

where $T_{k}=T S^{k}=N^{k} T$ and set $T_{0}=T$.

Proof. Straightforward.

Proposition 5.10. Let $(T, S, N)$ be a Kupershmidt-(dual-)Nijenhuis structure on a Malcev algebra $(A,[]$,$) with respect to a representation (V ; \varrho)$. Then all $T_{k}=N^{k} T$ are Kupershmidt operators with respect to the representation $(V ; \varrho)$ and for all $k, l \in \mathbb{N}, T_{k}$ and $T_{l}$ are compatible.

Proof. We only prove the conclusion for the Kupershmidt-Nijenhuis structure. The other one can be proved similarly.

By (5.3) and (5.6) with $i=0$, we have

$$
T_{k}[u, v]^{T_{k}}=\left[T_{k}(u), T_{k}(v)\right],
$$

which implies that $T_{k}$ is a Kupershmidt operator on $A$ with respect to a representation $(V ; \varrho)$.

For the second conclusion, we need to prove that $T^{k}+T^{k+i}$ is a Kupershmidt operator for all $k, i \in \mathbb{N}$. By (5.6), we have

$$
[u, v]^{T_{k}+T_{k+i}}=[u, v]^{T_{k}}+[u, v]^{T_{k+i}}=[u, v]^{T_{k}}+[u, v]_{S^{i}}^{T_{k}}
$$


Furthermore, we have

$$
\begin{aligned}
& \left(T_{k}+T_{k+i}\right)\left([u, v]^{T_{k}+T_{k+i}}\right) \\
= & T_{k}\left([u, v]^{T_{k}}\right)+T_{k}\left([u, v]_{S^{i}}^{T_{k}}\right)+T_{k+i}\left([u, v]^{T_{k}}\right)+T_{k+i}\left([u, v]_{S^{i}}^{T_{k}}\right) \\
= & T_{k}\left([u, v]^{T_{k}}\right)+T_{k+i}\left([u, v]_{S^{i}}^{T_{k}}\right)+T_{k+i}\left([u, v]_{S^{i}}^{T_{k}}\right) \\
& +T_{k}\left(\left[S^{i}(u), v\right]^{T_{k}}+\left[u, S^{i}(v)\right]^{T_{k}}-S^{i}[u, v]^{T_{k}}\right) \\
= & T_{k}\left([u, v]^{T_{k}}\right)+T_{k+i}\left([u, v]_{S^{i}}^{T_{k}}\right)+T^{k}\left(\left[S^{i}(u), v\right]^{T_{k}}\right)+T^{k}\left(\left[u, S^{i}(v)\right]^{T_{k}}\right) \\
= & {\left[T_{k}(u), T_{k}(v)\right]+\left[T_{k+i}(u), T_{k+i}(v)\right]+\left[T_{k+i}(u), T_{k}(v)\right]+\left[T_{k}(u), T_{k+i}(v)\right] } \\
= & {\left[\left(T_{k}+T_{k+i}\right)(u),\left(T_{k}+T_{k+i}\right)(v)\right] . }
\end{aligned}
$$

Thus $T^{k}+T^{k+i}$ is a Kupershmidt operator. The proof is finished.

Compatible Kupershmidt operators can give rise to Kupershmidt-dual-Nijenhuis structures.

Proposition 5.11. Let $T, T_{1}: V \longrightarrow A$ be two Kupershmidt operators on a Malcev algebra $(A,[]$,$) with respect to a representation (V ; \varrho)$. Suppose that $T$ is invertible. If $T$ and $T_{1}$ are compatible, then

(1) $\left(T, S=T^{-1} T_{1}, N=T_{1} T^{-1}\right)$ is a Kupershmidt-dual-Nijenhuis structure;

(2) $\left(T_{1}, S=T^{-1} T_{1}, N=T_{1} T^{-1}\right)$ is a Kupershmidt-dual-Nijenhuis structure.

Proof. Item 1. The proof of $(N, S)$ being a dual-Nijenhuis pair is similar to the proof of Theorem 4.8. We omit the details. It is obvious that $T S=N T$. Thus we only need to prove that the compatibility condition (4.9) holds. By the compatibility condition of $T$ and $T_{1}$ and Proposition 5.4, N=T $T_{1} T^{-1}$ is a Nijenhuis operator on the Malcev algebra $A$. By Proposition 5.2, we also have

$$
\begin{aligned}
{\left[T(u), T_{1}(v)\right]+\left[T_{1}(u), T(v)\right]=} & T\left(\varrho\left(T_{1}(u)\right)(v)-\varrho\left(T_{1}(v)\right)(u)\right) \\
& +T_{1}(\varrho(T(u))(v)-\varrho(T(v))(u)), \quad \forall u, v \in V .
\end{aligned}
$$

Substituting $T_{1}$ with $T S$, then we have

$$
\begin{aligned}
{[T(u), T S(v)]+[T S(u), T(v)]=} & T(\varrho(T S(u))(v)-\varrho(T S(v))(u)) \\
& +T S(\varrho(T(u))(v)-\varrho(T(v))(u)) .
\end{aligned}
$$

Since $T$ is a Kupershmidt operator on $A$ with respect to a representation $(V ; \varrho)$, we have

$$
\begin{aligned}
{[T(u), T S(v)]+[T S(u), T(v)]=} & T(\varrho(T(u))(S(v))-\varrho(T S(v))(u) \\
& +\varrho(T S(u))(v)-\varrho(T(v))(S(u))) .
\end{aligned}
$$

Since $T$ is invertible, (5.7) is equivalent to

$$
S(\varrho(T(u))(v)-\varrho(T(v))(u))=\varrho(T(u))(S(v))-\varrho(T(v))(S(u)) .
$$

On the other hand, we have

$$
[u, v]_{S}^{T}-[u, v]^{T S}=\varrho(T(u))(S(v))-\varrho(T(v))(S(u))-S(\varrho(T(u))(v)-\varrho(T(v))(u)) .
$$

Thus, (5.8) implies that $[u, v]_{S}^{T}=[u, v]^{T S}$. Therefore, $\left(T, S=T^{-1} T_{1}, N=T_{1} T^{-1}\right)$ is a Kupershmidt-dual-Nijenhuis structure. 
Item 2. By direct calculation, we have

$$
\begin{aligned}
& {[u, v]_{S}^{T_{1}}-[u, v]^{T_{1} S} } \\
= & \varrho\left(T_{1}(u)\right)(S(v))-\varrho\left(T_{1}(v)\right)(S(u))-S\left(\varrho\left(T_{1}(u)\right)(v)-\varrho\left(T_{1}(v)\right)(u)\right) \\
= & \varrho(T S(u))(S(v))-\varrho(T S(v))(S(u))-S(\varrho(T S(u))(v)-\varrho(T S(v))(u)) \\
= & {[S(u), S(v)]^{T}-S[u, v]^{T S}=0 . }
\end{aligned}
$$

Thus, $\left(T_{1}, S=T^{-1} T_{1}, N=T_{1} T^{-1}\right)$ is also a Kupershmidt-dual-Nijenhuis structure on $A$ with respect to a representation $(V ; \varrho)$.

\section{Infinitesimal deformations of a Kupershmidt operator of Malcev alge- bra}

In this section, we study infinitesimal deformations of a Kupershmidt operator of Malcev algebras. In particular, we introduce the notion of a Nijenhuis element associated to a Kupershmidt operator, which gives rise to a trivial infinitesimal deformation of the Kupershmidt operator. Their relationship with the infinitesimal deformations of the associated pre-Malcev algebra is also studied.

Definition 6.1. Let $T$ and $T^{\prime}$ be a two Kupershmidt operators on a Malcev algebra $(A,[]$,$) with respect to a representation (V ; \varrho)$. A morphism from $T^{\prime}$ to $T$ consists of a Malcev algebra morphism $\phi_{A}: A \longrightarrow A$ and a linear map $\varphi_{V}: V \longrightarrow V$ such that

$$
\begin{aligned}
T \circ \varphi_{V} & =\varphi_{A} \circ T^{\prime}, \\
\varphi_{V} \varrho(x)(u) & =\varrho\left(\varphi_{A}(x)\right)\left(\varphi_{V}(u)\right), \quad \forall x \in A, u \in V .
\end{aligned}
$$

In particular, if both $\varphi_{A}$ and $\varphi_{V}$ are invertible, $\left(\varphi_{A}, \varphi_{V}\right)$ is called an isomorphism from $T^{\prime}$ to $T$.

Proposition 6.2. Let $T$ and $T^{\prime}$ be two Kupershmidt operators on a Malcev algebra $(A,[]$, with respect to a representation $(V ; \varrho)$ and $\left(\varphi_{A}, \varphi_{V}\right)$ a morphism from $T^{\prime}$ to $T$. Then $\varphi_{V}$ is a morphism of pre-Malcev algebras from $\left(V,{ }^{T^{\prime}}\right)$ to $\left(V,{ }^{T}\right)$ defined in Theorem 4.3.

Proof. According to Eqs (6.1) and (6.2), for any $u, v \in V$, we have

$$
\begin{aligned}
\varphi_{V}\left(u \cdot T^{\prime} v\right) & =\varphi_{V} \varrho\left(T^{\prime} u\right)(v)=\varrho\left(\varphi_{A}\left(T^{\prime} u\right)\right)\left(\varphi_{V}(v)\right) \\
& =\varrho\left(T\left(\varphi_{V}(u)\right)\right)\left(\varphi_{V}(v)\right)=\varphi_{V}(u) \cdot_{T} \varphi_{V}(v) .
\end{aligned}
$$

Definition 6.3. Let $T$ be a Kupershmidt operator on a Malcev algebra $(A,[]$,$) with$ respect to a representation $(V ; \varrho)$ and $\mathcal{T}: V \longrightarrow A$ a linear map. If $T_{t}=T+t \mathcal{T}$ is still a Kupershmidt operator on the Malcev algebra $A$ with respect to the representation $(V ; \varrho)$ for all $t$, we say that $\mathcal{T}$ generates a one-parameter infinitesimal deformation of the Kupershmidt operator $T$.

By direct computation, we can check that $T_{t}=T+t \mathcal{T}$ is a one-parameter infinitesimal deformation of a Kupershmidt operator $T$ if and only if for any $u, v \in V$,

$$
\begin{aligned}
{[T u, \mathcal{T} v]+[\mathcal{T} u, T v] } & =T(\varrho(\mathcal{T} u)(v)-\varrho(\mathcal{T} v)(u))+\mathcal{T}(\varrho(T u)(v)-\varrho(T v)(u)), \\
{[\mathcal{T} u, \mathcal{T} v] } & =\mathcal{T}(\varrho(\mathcal{T} u)(v)-\varrho(\mathcal{T} v)(u)) .
\end{aligned}
$$

Note that Eq. (6.4) means that $\mathcal{T}$ is a Kupershmidt operator on the Malcev algebra $A$ associated to the representation $(V ; \varrho)$.

Now turning to a pre-Malcev algebra $(V, \cdot)$ given in Theorem 4.3, let $\psi: \otimes^{2} V \longrightarrow V$ be a linear map. If for any $t \in \mathbb{K}$, the multiplication ${ }_{t}$ defined by

$$
u \cdot{ }_{t} v:=u \cdot v+t \psi(u, v), \forall u, v \in V
$$


also gives a pre-Malcev algebra structure, we say that $\psi$ generates a one-parameter infinitesimal deformation of the pre-Malcev algebra $(V, \cdot)$.

The two types of infinitesimal deformations are related as follows.

Proposition 6.4. If $\mathcal{T}$ generates a one-parameter infinitesimal deformation of a Kupershmidt operator $T$ on a Malcev algebra $(A,[]$,$) with respect to a representation (V ; \varrho)$, then the product $\psi_{\mathcal{T}}$ on $V$ defined by

$$
\psi_{\mathcal{T}}(u, v):=\varrho(\mathcal{T} u)(v), \quad \forall u, v \in V
$$

generates a one-parameter infinitesimal deformation of the associated pre-Malcev algebra $\left(V,{ }^{T}\right)$.

Proof. Denote by ${ }_{t}$ the corresponding pre-Malcev algebra structure associated to the Kupershmidt operator $T+t \mathcal{T}$. Then we have

$$
u \cdot{ }_{t} v=\varrho((T+t \mathcal{T})(u))(v)=\varrho(T u)(v)+t \varrho(\mathcal{T} u)(v)=u \cdot{ }^{T} v+t \psi_{\mathcal{T}}(u, v), \forall u, v \in V,
$$

which implies that $\psi_{\mathcal{T}}$ generates a one-parameter infinitesimal deformation of $\left(V,{ }^{T}\right)$.

Corollary 6.5. If $\mathcal{T}$ generates a one-parameter infinitesimal deformation of a Kupershmidt operator $T$ on a Malcev algebra $(A,[]$,$) with respect to a representation (V ; \varrho)$. Then the product $\psi_{\mathcal{T}}$ on $V$ defined by

$$
\omega_{\mathcal{T}}(u, v):=\varrho(\mathcal{T} u)(v)-\varrho(\mathcal{T} v)(u), \quad \forall u, v \in V,
$$

generates a one-parameter infinitesimal deformation of the sub-adjacent Malcev algebra $\left(V,[\cdot, \cdot]^{T}\right)$ of the associated pre-Malcev algebra $\left(V, \cdot^{T}\right)$.

Definition 6.6. Let $T$ be a Kupershmidt operator on a Malcev algebra $(A,[]$,$) with$ respect to a representation $(V ; \varrho)$. Two one-parameter infinitesimal deformations $T_{t}^{1}=$ $T+t \mathcal{T}_{1}$ and $T_{t}^{2}=T+t \mathcal{T}_{2}$ are said to be equivalent if there exists an $x \in A$ such that $\left(\operatorname{Id}_{A}+\operatorname{tad}_{x}, \operatorname{Id}_{V}+t \varrho(x)\right)$ is a homomorphism from $T_{t}^{2}$ to $T_{t}^{1}$. In particular, a one-parameter infinitesimal deformation $T_{t}=T+t \mathcal{T}$ of an Kupershmidt operator $T$ is said to be trivial if there exists an $x \in A$ such that $\left(\operatorname{Id}_{A}+\operatorname{tad}_{x}, \operatorname{Id}_{V}+t \varrho(x)\right)$ is a homomorphism from $T_{t}$ to $T$.

Let $\left(\operatorname{Id}_{A}+\operatorname{tad} x, \operatorname{Id}_{V}+t \varrho(x)\right)$ be a homomorphism from $T_{t}^{2}$ to $T_{t}^{1}$. $\operatorname{Then} \operatorname{Id}_{A}+\operatorname{tad}$ is a Malcev algebra endomorphism of $A$. Thus, we have

$$
\left(\operatorname{Id}_{A}+\operatorname{tad}_{x}\right)[y, z]=\left[\left(\operatorname{Id}_{A}+\operatorname{tad}_{x}\right)(y),\left(\operatorname{Id}_{A}+\operatorname{tad}_{x}\right)(z)\right], \forall y, z \in A,
$$

which implies that $x$ satisfies

$$
[[x, y],[x, z]]=0, \quad \forall y, z \in A .
$$

Then by Eq. (6.1), we get

$$
\left(T+t \mathcal{T}_{1}\right)\left(\operatorname{Id}_{V}+t \varrho(x)\right)(u)=\left(\operatorname{Id}_{A}+\operatorname{tad}_{x}\right)\left(T+t \mathcal{T}_{2}\right)(u), \quad \forall u \in V,
$$

which implies

$$
\begin{aligned}
\left(\mathcal{T}_{2}-\mathcal{T}_{1}\right)(u) & =T \varrho(x)(u)+[T u, x], \\
\mathcal{T}_{1} \varrho(x)(u) & =\left[x, \mathcal{T}_{2} u\right], \forall u \in V .
\end{aligned}
$$

Finally, Eq. (6.2) gives

$$
\left(\operatorname{Id}_{V}+t \varrho(x)\right) \varrho(y)(u)=\varrho\left(\left(\operatorname{Id}_{A}+\operatorname{tad}_{x}\right)(y)\right)\left(\operatorname{Id}_{V}+t \varrho(x)\right)(u), \quad \forall y \in A, u \in V,
$$

which implies that $x$ satisfies

$$
\varrho([x, y]) \varrho(x)=0, \quad \forall y \in A .
$$


Definition 6.7. Let $T$ be a Kupershmidt operator on a Malcev algebra $A$ with respect to a representation $(V ; \varrho)$. An element $x \in A$ is called a Nijenhuis element associated to $T$ if $x$ satisfies Eqs. (6.5), (6.8) and the equation

$$
[x,[T u, x]+T \varrho(x)(u)]=0, \quad \forall u \in V .
$$

Denote by $N i j(T)$ the set of Nijenhuis elements associated to a Kupershmidt operator $T$.

By Eqs. (6.5)-(6.8), it is obvious that a trivial one-parameter infinitesimal deformation gives rise to a Nijenhuis element. The following result is in close analogue to the fact that the differential of a Nijenhuis operator on a Malcev algebra generates a trivial one-parameter infinitesimal deformation of the Malcev algebra, justifying the notion of Nijenhuis elements.

Theorem 6.8. Let $T$ be a Kupershmidt operator on a Malcev algebra $A$ with respect to a representation $(V ; \varrho)$. Then for any $x \in N i j(T), T_{t}:=T+t \mathcal{T}$ with $\mathcal{T}(u)=$ $T \varrho(x)(u)+[T u, x]$, for all $u \in V$, is a trivial one-parameter infinitesimal deformation of the Kupershmidt operator $T$.

Proof. First $\mathcal{T}$ is closed since $\mathcal{T}(u)=T \varrho(x)(u)+[T u, x]$. To show that $\mathcal{T}$ generates a trivial one-parameter infinitesimal deformation of the Kupershmidt operator $T$, we only need to verify that Eq. (6.4) holds. By Eq. (6.5), we have, for any $u, v \in V$,

$$
\begin{aligned}
& {[\mathcal{T} u, \mathcal{T} v]-\mathcal{T}(\varrho(\mathcal{T} u)(v)-\varrho(\mathcal{T} v)(u)) } \\
= & {[[T u, x],[T v, x]]+[[T u, x], T \varrho(x)(v)]+[T \varrho(x)(u),[T v, x]]+[T \varrho(x)(u), T \varrho(x)(v)] } \\
& -[T \varrho([T u, x])(v), x]-[T \varrho(T \varrho(x)(u))(v), x]+[T \varrho([T v, x])(u), x] \\
& +[T \varrho(T \varrho(x)(v))(u), x]-T \varrho(x) \varrho([T u, x])(v)-T \varrho(x) \varrho(T \varrho(x)(u))(v) \\
& +T \varrho(x) \varrho([T v, x])(u)+T \varrho(x) \varrho(T \varrho(x)(v))(u) \\
= & {[[T u, x], T \varrho(x)(v)]+[T \varrho(x)(u),[T v, x]]+T \varrho(T \varrho(x)(u)) \varrho(x)(v) } \\
& \underbrace{-T \varrho(T \varrho(x)(v)) \varrho(x)(u)}-[T \varrho([T u, x])(v), x]-[T \varrho(T \varrho(x)(u))(v), x] \\
& +[T \varrho([T v, x])(u), x]+[T \varrho(T \varrho(x)(v))(u), x] \\
& \frac{-T \varrho(x) \varrho([T u, x])(v)-T \varrho(x) \varrho(T \varrho(x)(u))(v)}{+T \varrho(x) \varrho([T v, x])(u)+T \varrho(x) \varrho(T \varrho(x)(v))(u)} .
\end{aligned}
$$

By Eqs. (6.8) and (6.9), the under-braced terms add to zero. Similarly, the underlined terms add to zero. For the other terms, by Eqs. (6.5) and (6.9), we have

$$
\begin{aligned}
& {[[T u, x], T \varrho(x)(v)]-[T \varrho([T u, x])(v), x]+[T \varrho(T \varrho(x)(v))(u), x] } \\
= & {[T u,[x, T \varrho(x)(v)]]+[[T u, T \varrho(x)(v)], x]-[T \varrho([T u, x])(v), x]+[T \varrho(T \varrho(x)(v))(u), x] } \\
= & -[T u,[x,[T v, x]]]+[T \varrho(T u) \varrho(x)(v), x]-[T \varrho([T u, x])(v), x] \\
= & -[x,[T u,[T v, x]]]+[T \varrho(x) \varrho(T u)(v), x]-[x,[T u,[T v, x]]] \\
= & +[x,[T \varrho(T u)(v), x]] .
\end{aligned}
$$

Similarly, we have

$$
\begin{aligned}
& {[T \varrho(x)(u),[T v, x]]-[T \varrho(T \varrho(x)(u))(v), x]+[T \varrho([T v, x])(u), x] } \\
= & {[x,[T v,[T u, x]]]-[x,[T \varrho(T v)(u), x]] . }
\end{aligned}
$$

Therefore,

$$
\begin{aligned}
& {[\mathcal{T} u, \mathcal{T} v]-\mathcal{T}(\varrho(\mathcal{T} u)(v)-\varrho(\mathcal{T} v)(u)) } \\
= & -[x,[T u,[T v, x]]]+[x,[T v,[T u, x]]]+[x,[T \varrho(T u)(v), x]]-[x,[T \varrho(T v)(u), x]] \\
= & -[x,[[T u, T v], x]]+[x,[[T u, T v], x]]=0,
\end{aligned}
$$

which means that $\mathcal{T}$ generates a one-parameter infinitesimal deformation of $T$. 
Further, since $x$ is a Nijenhuis element, it is straightforward to deduce that $\left(\operatorname{Id}_{A}+\right.$ $\left.\operatorname{tad}_{x}, \operatorname{Id}_{V}+t \varrho(x)\right)$ gives the desired homomorphism between $T_{t}$ and $T$. Thus, the deformation is trivial.

Now we introduce the notion of a Nijenhuis operator on a pre-Malcev algebra.

Definition 6.9. A linear map $N: A \longrightarrow A$ on a pre-Malcev algebra $(A, \cdot)$ is called a Nijenhuis operator if

$$
(N x) \cdot(N y)=N((N x) \cdot y+x \cdot(N y)-N(x \cdot y)), \quad \forall x, y \in A .
$$

For its connection with a Nijenhuis element associated to a Kupershmidt operator, we have the following proposition.

Proposition 6.10. Let $x \in A$ be a Nijenhuis element associated to an Kupershmidt operator $T$ on a Malcev algebra $A$ with respect to a representation $(V ; \varrho)$. Then $\varrho(x)$ is a Nijenhuis operator on the associated pre-Malcev algebra $\left(V,{ }_{T}\right)$.

Proof. For the proof, we just need to check, by Eq. (6.8), for all $u, v \in V$,

$$
\begin{aligned}
& \varrho(x)\left(\varrho(x)(u) \cdot \cdot_{T} v+u \cdot_{T} \varrho(x)(v)-\varrho(x)\left(u \cdot_{T} v\right)\right)-\varrho(x)(u) \cdot_{T} \varrho(x)(v) \\
= & \varrho(x)(\varrho(T \varrho(x)(u))(v)+\varrho(T u) \varrho(x)(v)-\varrho(x) \varrho(T u)(v))-\varrho(T \varrho(x)(u)) \varrho(x)(v) \\
= & {[\varrho(x), \varrho(T \varrho(x)(u))]+[\varrho(x), \varrho([T u, x])](v) } \\
= & \varrho([x, T \varrho(x)(u)+[T u, x]])(v)=0 .
\end{aligned}
$$

Acknowledgment. The author would like to thank the referee for valuable comments and suggestions on this article.

\section{References}

[1] C. Bai, L. Guo and X. Ni, Nonabelian generalized Lax pairs, the classical Yang-Baxter equation and PostMalcev algebras. Comm. Math. Phys. 297, 553-596, 2010.

[2] C. Bai, L. Guo and X. Ni, Generalizations of the classical Yang-Baxter equation and Kupershmidt operators. J. Math. Phys. 52, 063515, 2011.

[3] C. Bai, L. Guo and X. Ni, Relative Rota-Baxter operators and tridendriform algebras. J. Algebra Appl. 12, 5525-5537, 2013.

[4] D. Balavoine, Deformations of algebras over a quadratic Operads. Proceedings of Renaissance Conferences (Hartford, CT/Luminy, 1995), Contemp. Math. 202, 207$234,1997$.

[5] G. Baxter, An analytic problem whose solution follows from a simple algebraic identity, Pacific J. Math. 10, 731-742, 1960.

[6] D. Burde, Simple left-symmetric algebras with solvable Lie algebra, Manuscripta Math. 95, 397-411, 1998.

[7] J. Carinena, J. Grabowski and G. Marmo Quantum bi-Hamiltonian systems. Internat. J. Modern Phys. A 15 (30), 4797-4810, 2000.

[8] A. Connes and D. Kreimer, Renormalization in quantum field theory and the Riemann-Hilbert problem. I. The Hopf algebra structure of graphs and the main theorem, Comm. Math. Phys. 210, 249-273, 2000.

[9] I. Dorfman, Dirac structures and integrability of nonlinear evolution equations, Vol. 18. John Wiley \& Son Limited, 1993.

[10] T.F. Fox, An introduction to algebraic deformation theory. J. Pure Appl. Algebra 84, 17-41, 1993.

[11] M. Gerstenhaber, On the deformation of rings and algebras. Ann. of Math. 79, 59-103, 1964. 
[12] M. Gerstenhaber, On the deformation of rings and algebras. II. Ann. of Math. 84, $1-19,1966$.

[13] M. Gerstenhaber, On the deformation of rings and algebras. III. Ann. of Math. 88, 1-34, 1968.

[14] M. Gerstenhaber, On the deformation of rings and algebras. IV. Ann. of Math. 99, 257-276, 1974.

[15] L. Guo, An introduction to Rota-Baxter algebra. Surveys of modern mathematics, 4. International Press,Vol. 2. No.9. p.14. Somerville, MA; Higher Education Press, Beijing, 2012.

[16] F. Gürsey and C.H. Tze, On the role of division, Jordan and related algebras in particle physics, World Scientific, Singapore, 1996.

[17] F. S. Kerdman, Analytic Moufang loops in the large, Alg. Logic 18 , 325-347, 1980.

[18] M. Kontsevich and Y. Soibelman, Deformation theory. I [Draft], http://www.math.ksu.edu/ soibel/Book-vol1.ps, 2010.

[19] B. A. Kupershmidt, What a classical r-matrix really is, J. Nonlinear Math. Phy. 6, 448-488, 1999.

[20] E.N. Kuz'min, The connection between Mal'cev algebras and analytic Moufang loops, Alg. Logic 10, 1-14, 1971.

[21] J.L. Loday and B. Vallette, Algebraic operads, Springer, 2012.

[22] S. Madariaga, Splitting of operations for alternative and Malcev structures. Communications in Algebra, 45 (1), 183-197, 2014.

[23] A.I. Mal'tsev, Analytic loops, Mat. Sb. 36, 569-576, 1955.

[24] H.C. Myung, Malcev-admissible algebras, Progress in Math. Vol. 64, Birkhäuser, Boston, MA, 1986.

[25] P.T. Nagy, Moufang loops and Malcev algebras, Sem. Sophus Lie 3, 65-68, 1993.

[26] A. Nijenhuis and R. Richardson, Cohomology and deformations in graded Lie algebras. Bull. Amer. Math. Soc. 72, 1-29, 1966.

[27] A. Nijenhuis and R. W. Richardson, Deformation of Malcev algebra structures, J. Math. Mech. 17, 89-105, 1967.

[28] A. Nijenhuis and R. Richardson, Commutative algebra cohomology and deformations of Lie and associative algebras. J. Algebra 9, 42-105, 1968.

[29] S. Okubo, Introduction to octonions and other non-associative algebras in physics, Cambridge Univ. Press, Cambridge, UK, 1995.

[30] A. Sagle, Malcev algebras, Trans. Amer. Math. Soc. 101, 426-458, 1961.

[31] L.V. Sabinin, Smooth quasigroups and loops, Kluwer Academic, The Netherlands, 1999.

[32] K. Uchino, Quantum Analogy of Poisson Geometry, Related Dendriform Algebras and Rota-Baxter Operators, Lett. Math. Phys. 85, 91-109, 2008.

[33] K. Uchino, Twisting on associative algebras and Rota-Baxter type operators. J. Noncommut. Geom. 4, 349-379, 2010. 